\title{
BMJ Open Postneoadjuvant surveillance and surgery as needed compared with postneoadjuvant surgery on principle in multimodal treatment for oesophageal cancer: a scoping review protocol
}

\author{
Christine Schmucker (D) , ${ }^{1}$ Blin Nagavci, ${ }^{1}$ Julian Hipp (D) , ${ }^{2}$ Claudia Schmoor (D) , ${ }^{3}$ \\ Joerg Meerpohl, ${ }^{1,4}$ Jens Hoeppner (i) ${ }^{2,5}$
}

To cite: Schmucker C, Nagavci B, Hipp J, et al. Postneoadjuvant surveillance and surgery as needed compared with postneoadjuvant surgery on principle in multimodal treatment for oesophageal cancer: a scoping review protocol. BMJ Open 2021;11:e044190. doi:10.1136/ bmjopen-2020-044190

- Prepublication history for this paper is available online. To view these files, please visit the journal online (http://dx.doi. org/10.1136/bmjopen-2020044190).

Received 28 August 2020 Revised 21 December 2020 Accepted 08 January 2021

Check for updates

(C) Author(s) (or their employer(s)) 2021. Re-use permitted under CC BY-NC. No commercial re-use. See rights and permissions. Published by BMJ.

For numbered affiliations see end of article.

Correspondence to Prof Dr Jens Hoeppner; jens.hoeppner@uksh.de

\section{ABSTRACT}

Introduction In current medical practice of curative treatment for non-metastatic oesophageal cancer, surgery on principle is carried out by oesophagectomy after neoadjuvant treatment. However, oesophagectomy is often associated with postoperative morbidity and mortality. Taking into account that modern neoadjuvant therapy is effective and many of patients show no vital tumour cells in the operative specimens, we aim to perform a scoping review as part of the development phase for a prospectively planned multicentre randomised controlled trial investigating 'surgery as needed vs surgery on principle in patients with postneoadjuvant complete response of oesophageal cancer' (Prospective trial registration number DRKS00022801). This scoping approach will allow us to finally define and/or adapt the research question including the design and methodology of the randomised controlled trial taking into account the findings for example, research gaps and/or pitfalls in the currently available study pool addressing this or very similar questions.

Methods and analysis To identify relevant research, we will conduct searches in the electronic databases Medline, Web of Science Core Collection, Cochrane Library and Science Direct. We will also check references of relevant studies and perform a cited reference research (forward citation tracking). Titles and abstracts of the records identified by the searches will be screened and full texts of all potentially relevant articles will be obtained. We will consider randomised trials and non-randomised controlled studies. Data extraction tables will be set up, including study and patients' characteristics, aim of study and reported outcomes. We will summarise the data using tables and figures (eg, bubble plots) to present the research landscape and to describe potential clusters and/or gaps to support the planning of a randomised trial in this patient population. Ethics and dissemination Ethical approval is not required for this scoping review. Study findings will be shared by publication in a peer-reviewed journal and by presentation to key stakeholders on scientific meetings.

\section{INTRODUCTION}

Neoadjuvant chemoradiation (nCRT) and neoadjuvant chemotherapy (nCTX)
Strengths and limitations of this study

- The scoping review is part of the development phase for a prospectively planned multicentre randomised trial, addressing 'Surgery as needed versus surgery on principle in patients with post-neoadjuvant complete response of oesophageal cancer' (Prospective trial registration number DRKS00022801)

- The scoping review will allow us to finally define and/or adapt the research question, the design and methodology of the following randomised trial.

- The scoping review protocol is guided by validated methodological frameworks and the scoping review will be reported according to the preferred reporting items for systematic review and meta-analysis statement for scoping reviews, and therefore, will be conducted in line with 'the state-of-the-art' criteria.

- We will conduct comprehensive literature searches and map the current ongoing and published studies by extracting and cluster key data such as neoadjuvant treatment protocols, diagnostic methods of response evaluation and surveillance, and therapeutic outcomes.

- The final scoping review will be limited to English and German language studies.

improve patients' survival in curative treatment of non-metastatic oesophageal cancer and have become the standard of care in Western Europe. ${ }^{1}$ In these multimodal oncological protocols, curative surgery is carried out after neoadjuvant treatment by oesophagectomy. However, oesophagectomy implicates postoperative mortality rates between $6 \%$ and $11 \%$ and postoperative morbidity rates range from $60 \%$ to $80 \% \cdot{ }^{2-4}$ In recent years, neoadjuvant therapy has become increasingly effective, with $16 \%-49 \%$ of patients showing no tumour cells in the operative specimens. ${ }^{5-7}$ This high locoregional histopathological 
complete response rate imposes a need to identify complete responder and avoid potentially unnecessary and harmful surgery in this population. Considering that neoadjuvant treatment without surgery is effective for a large proportion of patients, more individual/personalised treatment options based on surveillance and surgery only if needed are highly relevant for patients with non-metastatic oesophageal cancer.

\section{OBJECTIVES}

We aim to perform a scoping review as part of the development phase for a prospectively planned multicentre randomised controlled trial, addressing 'Surgery as needed vs surgery on principle in patients with post-neoadjuvant complete response of oesophageal cancer' (Prospective registration identifier of the clinical trial will be DRKS00022801. Registration is currently in process and will be completed after we have incoperated the results of the scoping review). The scoping review will allow us to finally define and adapt the research question and methodology of the following randomised trial taking into account the findings (such as research gaps and/or methodological pitfalls) in the currently available pool of primary studies addressing this or very similar questions.

The objectives of the scoping review are as follows:

1. What specific neoadjuvant protocols of nCRT and nCTX have been studied for surveillance and surgery as needed?

2. In what populations or settings have these protocols been studied?

3. Which diagnostic methods have been used for postneoadjuvant tumour staging and surveillance of tumour response?

4. Which outcomes have been addressed in the clinical studies on surveillance and surgery as needed in oesophageal cancer?

5. What results were observed with respect to survival rates?

\section{METHODS AND ANALYSIS}

This protocol is written with reference to the preferred reporting items for systematic review and meta-analysis protocols statement ${ }^{8}$ and 'a priori' defines the methodology on which the scoping review will be based on:

\section{Eligibility criteria}

Participants/population

We will focus on studies including adults with nonmetastatic oesophageal cancer (after receiving neoadjuvant treatment). Studies including patients with distant metastases of oesophageal cancer, presence of gastric cancer; and/or participants younger than 18 years of age will be excluded.

\section{Box 1 Outcome variables.}

Outcomes (list will be completed depending on outcomes reported in the available study pool)

- Overall survival.

- Progression-free survival.

- Proportion of radical resection margin.

- Postoperative complications. (frequency and severity).

- Rate and timing of distant dissemination.

Disease recurrence rate.

Intervention and comparator treatment

We will consider surveillance after neoadjuvant therapy as eligible intervention. Surgery on principle after neoadjuvant therapy will be the comparator treatment.

\section{Context}

We will consider all nCTX and nCRT interventions implemented and evaluated in the context of non-metastatic oesophageal cancer.

\section{Relevant outcomes}

We will capture any outcomes reported in the eligible study pool. Highly important outcomes are displayed in box 1 . This table is non-exhaustive and will be completed depending on the outcomes reported in the identified study pool.

\section{Study types}

Randomised controlled trials; non-randomised controlled studies (using strategies of non-random allocation for assigning interventions) and observational studies (with control group) will be eligible for the scoping review. We will not consider single arm studies. Due to a missing control group within this study design. The reason for this exclusion is that studies without a control group provide no reliable data to estimate comparative effectiveness and will, therefore, not be useful for the planned randomised trial. Furthermore, review articles, clinical guidelines and work that has not been peer-reviewed (eg, thesis, editorials, letters, comments) will be excluded.

We will not apply any exclusion criteria regarding study duration and/or the study setting.

\section{Information sources}

The searches for this scoping review will be performed and conducted by following the recommendation of PRESS (Peer Review of Electronic Search Strategies) ${ }^{9}$; that is, a medical sciences librarian will develop the search strategies; in addition, search strategies will be validated by checking whether they identified studies already known. We will not use any date restrictions in the electronic searches. For each database, the date of the search, the search strategy and the number of search results will be documented.

Systematic searches for relevant published trials will be conducted in the following electronic data sources 
Table 1 Preliminary search strategy for Medline (Ovid).

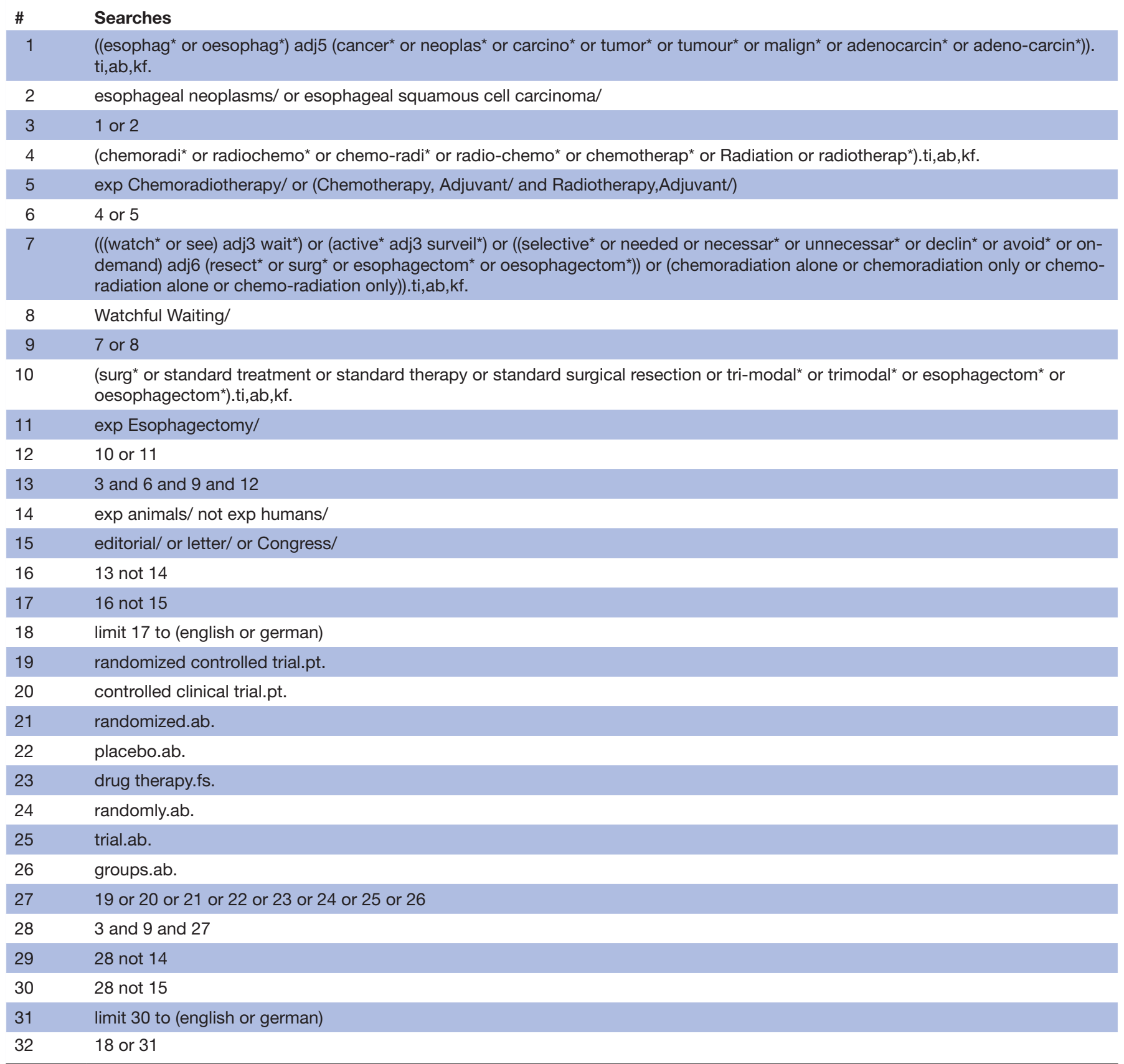

- Medline, Medline Daily Update, Medline In Process \& Other Non-Indexed Citations, Medline Epub Ahead of Print (via Ovid) (a preliminary search strategy is displayed in table 1).

- Web of Science Core Collection: Science Citation Index-EXPANDED (SCI-EXPANDED) (via Clarivate Analytics).

- Cochrane Library (via Wiley).

- Science Direct (via Elsevier).

Searches for unpublished and ongoing studies will be performed in ClinicalTrials.gov (www.clinicaltrials.gov), WHO International Clinical Trials Registry Platform (http://www.who.int/ictrp/search/en) and the German study register (www.drks.de).
We will use relevant studies and/or systematic reviews to search for additional references via the PubMed similar articles function (https://www.nlm.nih.gov/bsd/disted/ pubmedtutorial/020_190.html), and forward citation tracking. Reference lists of relevant studies and systematic reviews will also be reviewed manually.

\section{Identification of relevant Studies}

Titles and abstracts of the records identified by the searches will be screened and full texts of all potentially relevant articles will be obtained. Full texts will be checked for eligibility, by two reviewers and reasons for exclusions will be documented (full-text screening). The 
complete screening process will be conducted in Covidence (https://www.covidence.org).

\section{Extraction of study data/data items}

The following study data will be extracted and relevant information tabulated and/or described descriptively:

- Study characteristics, that is, author, year of publication, study type (randomised trial, non-randomised study) and design (superiority, non-inferiority trial), study status (eg, planned, ongoing, completed, prematurely discontinued), start and end of study.

- Details regarding sample size calculation.

- Details on sample size (number of participants screened and randomised/finally included; reasons for screening failures and number and reasons for drop-offs and compliance).

- Aim of the study.

- Setting, that is, geographical and organisational setting.

- Characteristics and definition of participants (age, gender, tumour histology and tumour stage).

- Details on neoadjuvant therapy (drug names, dose).

- Details on the diagnostic methods used for postneoadjuvant tumour staging and surveillance of tumour response.

- Definition of complete responders.

- Characteristics of intervention/surveillance group (definition of surveillance).

- Characteristics of comparator/surgery group (type of surgery and time between neoadjuvant therapy and surgery).

- Pathohistological complete response rate after neoadjuvant therapy (surgery group).

- On-demand surgery rate (surveillance group).

- All reported outcomes and their exact definitions, that is, how and when the outcome measures were assessed.

- Recruitment and follow-up time (planned and actual time).

Data from each included study will be extracted by one reviewer and checked by a second. Disagreements will be resolved through discussion until consensus will be reached.

\section{Risk of bias}

Risk of bias assessment is not part of a scoping review and will not be assessed accordingly. ${ }^{10} 11$

The methodology of the scoping review may be adapted minimally during the review process itself in terms of eligibility criteria, data extraction and outcome variables. ${ }^{12} 13$

\section{Data analyses/summary}

We will summarise the collected study data using tables and figures (eg, bubble plots) to present the research landscape and to describe potential clusters and/or gaps to support the planning of the proposed randomised trial in this patient population.

\section{Perspective/discussion}

Currently in Western Europe the majority of patients with non-metastatic resectable oesophageal cancer are treated with nCTX or nCRT plus consecutive surgery. Despite postneoadjuvant pathological complete response rates between $16 \%$ and $49 \%$, surgery is carried out in all patients and independent of the results of postneoadjuvant response evaluation. ${ }^{5-7}$ The 'Nationale Dekade gegen Krebs' programme of the german national government (https://www.dekade-gegen-krebs.de/de/ praxisveraendernde-studien-fuer-eine-bessere-patientenversorgung-2018.html) is supporting a multicentre randomised trial (which will be conducted by our study group) challenging this 'sometimes potentially harmful' algorithm by comparing postneoadjuvant surgery on principle versus surveillance (with surgery only if needed in the event of a persisting or recurring local tumour). Using a randomised study design, we aim to optimise therapeutic outcomes by personalisation of the therapeutic sequence. According to the current evidence and also supported by our clinical experience, it is likely that a subgroup of pathological complete responders (with consecutive omission of potentially harmful surgery) will be identified. ${ }^{14}$ A survival disadvantage of delayed surgery in case of local tumour relapse is likely to be excluded in a protocol of close surveillance in complete responder. ${ }^{15}$

Although the scoping review may not provide effect estimates including an evaluation of the certainty of evidence, it will be of great value to crystallise research questions, and the extent of available evidence by highlighting areas where evidence is lacking. The scoping review will support us to map the existing primary research for potential duplications. Furthermore, it will provide an overview of the (1) characteristics and definitions of patient populations (included in available studies) and settings, (2) details on the interventions (including type of neoadjuvant therapy, time between neoadjuvant therapy and surgery, definition of surveillance), (3) details on the diagnostic methods used for postneoadjuvant tumour staging, (4) definition of complete responders, (5) outcome measures and (6) follow-up times. Hence the scoping process will allow us to systematically develop the concept of the randomised trial based on current knowledge (including pitfalls) in this newly emerging treatment area.

By searching the searching the literature in different databases (ie, behind Medline) and also study registers (eg, ClinicalTrials.gov), all relevant completed but also ongoing studies comparing surveillance with surgery on demand in esophaegeal cancer will be identified. Finally the results of the scoping review will reveal (1) whether the diagnostic methods used and the definition for complete responders were appropriate and homogeneous, (2) whether the included sample sizes were sufficient to draw conclusions on benefits and harms, (3) what interventions were considered (eg, nCRT and/or chemotherapeutic protocols), (4) what outcomes of interest were covered, (5) whether follow-up times were sufficient and (6) whether clinical results across studies are 
homogeneous. We believe that the planned randomised trial will benefit from this state-of-the art research approach, and therefore, will provide patients, clinicians and other stakeholders with high evidence considering various patient-relevant outcomes when comparing these two treatment approaches.

Furthermore, parallel to the scoping review patient's values and perspectives towards choice of treatment will be analysed (DRKS00022050) prior to the start of the randomised trial and patient oriented information material for the trial will be developed and provided.

Overall, the final goal will be the development and verification of a protocol to identify patients with pathological complete response (based on reliable diagnostic methods and definitions for complete responders) who would not need to undergo high-risk surgery in the increasing subgroup of postneoadjuvant complete responders. This treatment procedure is expected to reduce morbidity and mortality rates, and increase quality of life. Regarding the socioeconomic impact, omission of oesophagectomy reduces treatment duration, complication rates and time of hospital stay. This results in reduced treatment costs and a faster return to normal life for this patient population.

\section{ETHICS AND DISSEMINATION}

Formal ethical approval is not required, as primary patient data will not be collected in this scoping review. We plan to publish the full scoping review in a peer-reviewed journal and to present the results at national and international scientific conferences.

\section{Author affiliations}

${ }^{1}$ Institute for Evidence in Medicine (for Cochrane Germany Foundation), Medical Center - University of Freiburg, Faculty of Medicine, University of Freiburg, Freiburg, Baden-Württemberg, Germany

${ }^{2}$ Center for Surgery, Department of General and Visceral Surgery, Medical Center - University of Freiburg, Faculty of Medicine, University of Freiburg, Freiburg, BadenWürttemberg, Germany

${ }^{3}$ Clinical Trials Unit, Medical Center - University of Freiburg, Faculty of Medicine, University of Freiburg, Freiburg im Breisgau, Baden-Württemberg, Germany ${ }^{4}$ Cochrane Germany, Cochrane Germany Foundation, Freiburg, Germany ${ }^{5}$ Department of Surgery, University Medical Center Schleswig-Holstein, Campus Luebeck, Luebeck, Germany

Contributors All authors of the manuscript meet the ICMJE criteria for authorship: $\mathrm{ChS}, \mathrm{BN}$ and JM designed the scoping review protocol. ChS and BN designed the preliminary search strategy. JeH and JuH contributed as experts for surgical and multimodal treatment of oesophageal cancer and provided scientific knowledge. $\mathrm{ChS}$ and $\mathrm{JH}$ wrote the scoping review protocol. JuH and CS are guarantors of the manuscript.

Funding This work was supported by a research grant (grand number 01KD1908) 'Nationale Dekade gegen Krebs' provided to JeH by the federal ministry of education and research (BMBF).

Disclaimer The funder was not involved in the development of the protocol. The article processing charge was funded by the Baden-Wuerttemberg Ministry of Science, Research and Art and the University of Freiburg in the funding programme Open Access Publishing.

Competing interests None declared.
Patient and public involvement Patients and/or the public were involved in the design, or conduct, or reporting, or dissemination plans of this research. Refer to the Methods section for further details.

Patient consent for publication Not required.

Provenance and peer review Not commissioned; externally peer reviewed.

Open access This is an open access article distributed in accordance with the Creative Commons Attribution Non Commercial (CC BY-NC 4.0) license, which permits others to distribute, remix, adapt, build upon this work non-commercially, and license their derivative works on different terms, provided the original work is properly cited, appropriate credit is given, any changes made indicated, and the use is non-commercial. See: http://creativecommons.org/licenses/by-nc/4.0/.

\section{ORCID iDs}

Christine Schmucker http://orcid.org/0000-0002-1188-3158

Julian Hipp http://orcid.org/0000-0001-9291-9695

Claudia Schmoor http://orcid.org/0000-0001-5610-9425

Jens Hoeppner http://orcid.org/0000-0002-3485-7446

\section{REFERENCES}

1 Hoeppner J, Zirlik K, Brunner T, et al. Multimodal treatment of locally advanced esophageal adenocarcinoma: which regimen should we choose? Outcome analysis of perioperative chemotherapy versus neoadjuvant chemoradiation in 105 patients. J Surg Oncol 2014;109:287-93.

2 Nimptsch U, Mansky T. Hospital volume and mortality for 25 types of inpatient treatment in German hospitals: observational study using complete national data from 2009 to 2014. BMJ Open 2017; 7:e016184.

3 Taioli E, Schwartz RM, Lieberman-Cribbin W, et al. Quality of life after open or minimally invasive esophagectomy in patients with esophageal cancer-a systematic review. Semin Thorac Cardiovasc Surg 2017;29:377-90.

4 van der Sluis PC, van der Horst S, May AM, et al. Robot-Assisted minimally invasive Thoracolaparoscopic esophagectomy versus open transthoracic esophagectomy for resectable esophageal cancer: a randomized controlled trial. Ann Surg 2019;269:621-30.

5 Al-Batran S-E, Hofheinz RD, Pauligk C, et al. Histopathological regression after neoadjuvant docetaxel, oxaliplatin, fluorouracil, and leucovorin versus epirubicin, cisplatin, and fluorouracil or capecitabine in patients with resectable gastric or gastrooesophageal junction adenocarcinoma (FLOT4-AIO): results from the phase 2 part of a multicentre, open-label, randomised phase $2 / 3$ trial. Lancet Oncol 2016;17:1697-708.

6 Homann N, Pauligk C, Luley K, et al. Pathological complete remission in patients with oesophagogastric cancer receiving preoperative 5-fluorouracil, oxaliplatin and docetaxel. Int J Cancer 2012;130:1706-13.

7 van Hagen P, Hulshof MCCM, van Lanschot JJB, et al. Preoperative chemoradiotherapy for esophageal or junctional cancer. $N$ Engl J Med 2012;366:2074-84.

8 Moher D, Shamseer L, Clarke M, et al. Preferred reporting items for systematic review and meta-analysis protocols (PRISMA-P) 2015 statement. Syst Rev 2015;4:1.

9 McGowan J, Sampson M, Salzwedel DM, et al. PRESS Peer Review of Electronic Search Strategies: 2015 Guideline Statement. J Clin Epidemiol 2016;75:40-6.

10 Grant MJ, Booth A. A typology of reviews: an analysis of 14 review types and associated methodologies. Health Info Libr J 2009;26:91-108.

11 Higgins JPT, Altman DG, Sterne JAC. Chapter 8: Assessing risk of bias in included studies. In: Higgins JPT, Churchill R, Chandler $\mathrm{J}$, et al, eds. Cochrane Handbook for systematic reviews of Interventionsversion 5.2.0. (updated June2017), Cochrane, 2017. www.training.cochrane.org/handbook

12 Levac D, Colquhoun H, O'Brien KK. Scoping studies: advancing the methodology. Implement Sci 2010;5:69.

13 Arksey H, O'Malley L. Scoping studies: towards a methodological framework. Int J Soc Res Methodol 2005;8:19-32.

14 Noordman BJ, Spaander MCW, Valkema R, et al. Detection of residual disease after neoadjuvant chemoradiotherapy for oesophageal cancer (preSANO): a prospective multicentre, diagnostic cohort study. Lancet Oncol 2018;19:965-74.

15 Taketa T, Xiao L, Sudo K, et al. Propensity-based matching between esophagogastric cancer patients who had surgery and who declined surgery after preoperative chemoradiation. Oncology 2013;85:95-9. 